\title{
Erratum
}

\section{Caffeoylquinic Acids in Centella asiatica Protect against Amyloid- $\beta$ Toxicity}

Nora E. Gray, Jeff Morré, Jeremiah Kelley, Claudia S. Maier, Jan F. Stevens, Joseph F. Quinn and Amala Soumyanath

[Journal of Alzheimer's Disease 40(2) (2014), 359-373, DOI 10.3233/JAD-131913]

http://iospress.metapress.com/content/y1n86017401841q7/

On page 371, in the Acknowledgment, 2nd line:

The grant number was incorrectly given as P50 AT00066. The current grant number from for A. Soumyanath from the NIH should be 3P30 AG000817 24 S1. 